\title{
Evidence of Antibody Repertoire Functional Convergence through Public Baseline and Shared Response Structures
}

\author{
Matthew I. J. Raybould ${ }^{1}$, Claire Marks ${ }^{1}$, Aleksandr Kovaltsuk ${ }^{1}$, Alan P. Lewis ${ }^{2}$, Jiye Shi ${ }^{3}$, and Charlotte M. Deane ${ }^{1 凶}$ \\ ${ }^{1}$ Oxford Protein Informatics Group, Department of Statistics, University of Oxford, 24-29 St Giles', Oxford, OX1 3LB, UK \\ ${ }^{2}$ Data and Computational Sciences, GlaxoSmithKline Research and Development, Gunnels Wood Road, Stevenage, SG1 2NY, UK \\ ${ }^{3}$ Chemistry Department, UCB Pharma, 216 Bath Road, Slough, SL1 3WE, UK
}

\begin{abstract}
The antibody repertoires of different individuals ought to exhibit significant functional commonality, given that most pathogens trigger a successful immune response in most people. Sequence-based approaches have so far offered little evidence for this phenomenon. For example, a recent study estimated the number of shared ('public') antibody clonotypes in circulating baseline repertoires to be around $0.02 \%$ across ten unrelated individuals. However, to engage the same epitope, antibodies only require a similar binding site structure and the presence of key paratope interactions, which can occur even when their sequences are dissimilar. Here, we investigate functional convergence in human antibody repertoires by comparing the antibody structures they contain. We first structurally profile baseline antibody diversity (using snapshots from 41 unrelated individuals), predicting all modellable distinct structures within each repertoire. This analysis uncovers a high (much greater than random) degree of structural commonality. For instance, around $3 \%$ of distinct structures are common to the ten most diverse individual samples ('Public Baseline' structures). Our approach is the first computational method to provide support for the long-assumed levels of baseline repertoire functional commonality. We then apply the same structural profiling approach to repertoire snapshots from three individuals before and after flu vaccination, detecting a convergent structural drift indicative of recognising similar epitopes ('Public Response' structures). Antibody Model Libraries derived from Public Baseline and Public Response structures represent a powerful geometric basis set of low-immunogenicity candidates exploitable for general or target-focused therapeutic antibody screening.
\end{abstract}

antibody repertoire | structural profiling | public structures | antibody model libraries | antibody screening

Correspondence: deane@stats.ox.ac.uk

\section{Introduction}

A key component of the human immune system is the antibody/B-cell receptor (BCR) repertoire, a diverse array of immunoglobulins tasked with identifying pathogens and initiating the adaptive immune response. Broad pathogenic recognition is achieved through enormous variable domain sequence diversity, with an estimated $10^{10}$ unique heavy variable domains $(\mathrm{VH})$ circulating at any one time from a theoretical set of $10^{12}$ (or $10^{16}-10^{18}$ full antibodies if light variable domain (VL) combinations are considered (1)).

On antigenic exposure, 'baseline' (resting-state) antibodies with sufficiently complementary binding sites to an antigen surface epitope are positively selected. The corresponding parent B cells subsequently migrate to the marginal zone of the lymph nodes, where intentional mutations are introduced to their sequence and only the highest-affinity binders survive in the competition for cognate T-helper cells (2).

Therefore, sequencing antibody repertoires before and during an immune response (e.g. vaccination) can reveal how different people respond to the same antigenic challenge, and can both improve our understanding of immunology and inform future vaccine or therapeutic design (3-5). Similarly, comparing the repertoires of healthy individuals against immunosuppressed (e.g. HIV) patients may also make known the origins of increased disease susceptibility (6-8).

However, sequencing an entire antibody repertoire is challenging; they are so large that conventional sequencing techniques, such as Sanger sequencing, do not capture enough of the diversity to be informative. Instead, high-throughput immunoglobulin gene sequencing (Ig-seq) technologies (e.g. Illumina MiSeq) are used. These methods create snapshots that are typically on the order of $10^{6}-10^{7} \mathrm{VH}$ and/or VL (unpaired) chains, up to a recent upper bound of around $10^{9}$ $(1,9,10)$. Single-cell sequencing methods, capable of preserving VH-VL chain pairings, are now emerging, however their current throughput yields datasets that are too small to study entire repertoire diversity (11-13).

Since most publicly-available Ig-seq data covers only the VH domain, the vast majority of whole-repertoire analysis has been performed over this region alone. The primary analytical method is currently 'clonotyping' (14-16). Clonotyping is a computational technique used to sort sequencing datasets into sets of functionally similar chains based on sequence features, and can be performed in several ways. The most common implementation groups sequences with the same predicted $\mathrm{V}$ and $\mathrm{J}$ gene transcript origins and above a certain percentage (same length) Complementarity-Determining Region H3 (CDRH3) sequence identity.

Such sequence-based approaches have contributed significantly to our knowledge of core immunology. For example, to estimate the true level of sequence similarity that exists across individuals, Briney et al. performed deep sequencing and clonotyping of the circulating baseline $\mathrm{VH}$ repertoires of ten volunteers (1). They found that just $0.022 \%$ of observed clonotypes were 'public' (seen in everyone). In a comple- 
mentary approach, Greiff et al. trained a Support Vector Machine on public and private clonal sequences to identify their high-dimensional features, proving that they have distinct immunogenomic properties (17).

Clonotyping can also be used to detect antigen-specific immunoglobulins, through the identification of expanded clones after vaccination, or those present in unusually high proportions in individuals immune to certain diseases. Explorations of expanded lineages have yielded high-affinity antibodies and $T$ cells against numerous pharmacologically interesting antigens, such as HIV proteins (6), cluster of differentiation proteins (18), botulinum neurotoxin serotype A (19), proteins implicated in type-1 diabetes (20), and many more.

However, clonotyping is only likely to identify a small subset of the true number of functionally equivalent antibodies. This is because it assumes that antibodies require a similar genetic background and high CDRH3 sequence identity to achieve complementarity to the same epitope. In reality, similar binding site structures and paratopes can be achieved from different genetic origins (21) and with surprisingly low CDRH3 sequence identity (22). It is also the case that not every epitope is naturally suited to CDRH3-dominated binding, instead preferring broader engagement by multiple CDRs (23). It is difficult to capture these functionally equivalent antibodies by sequence alone. An alternative approach would be to compare the three-dimensional structures of the antibodies, as binders to a given epitope are likely to adopt a similar geometry with residues capable of recapitulating key binding interactions at equivalent topological locations.

Experimental structure determination (e.g. by X-ray crystallography) remains too slow to solve representative portions of antibody repertoires (24). However, structural annotation approaches are now fast enough to geometrically characterise the individual CDRs of millions of sequences a day with increasing accuracy $(25,26)$. So far, these analyses have focussed solely on the VH chain, and none have considered the impact of VL on binding site configuration. This can most accurately be captured through variable domain (Fv) modelling, and recent developments have afforded homology approaches with sufficient throughput to analyse meaningful portions of the repertoire $(27,28)$. For example, a recent prototype structural profiling method that creates representative Fv model libraries from large repertoire snapshots, with applications in developability issue prediction (29).

In this paper, we further refine this repertoire structural profiler, and apply the optimised pipeline to the task of repertoire functional screening. We first analyse 41 baseline antibody repertoires from unrelated individuals, and find that the same representative ('distinct') binding site structures are predicted to appear across many individuals ('Public Baseline' structures). We also show, through the construction of 'Random Repertoires', that this level of structural sharing is far greater than would be expected by chance. Our data therefore represents the first computational evidence that sizeable functional commonality could exist in the baseline repertoires of different people. We then implement the same pipeline on pre- and post-vaccination datasets from three unrelated in- dividuals, detecting a significant increase in structural commonality, and identifying all convergent response structures that may recognise similar epitopes ('Public Response' structures). We built Antibody Model Libraries (AMLs) by homology modelling a VH-VL sequence pairing predicted to adopt each Public Baseline or Public Response structure. In silico analysis of these AMLs shows that they represent a powerful geometric basis set of low-immunogenicity candidates exploitable for general or target-focused therapeutic antibody screening.

\section{Results}

This study comprises two main investigations. First, we use data from an immunoglobulin gene sequencing (Ig-seq) study by Gidoni et al. (30) to investigate the degree of structural overlap in the circulating baseline repertoires of many unrelated individuals. We then use data from a longitudinal Ig-seq flu vaccination study by Gupta et al. (5) to measure three individuals' structural responses to exposure to a common antigen. Both translated Ig-seq datasets were downloaded from the Observed Antibody Space (OAS) database (9), retaining only the 41 Gidoni volunteers with sufficiently deep reads (see Methods).

We used an updated version of our repertoire structural profiling pipeline (29) for improved accuracy in CDR structure and VH-VL interface orientation prediction (see Methods, SI Fig. 4). Briefly, repertoire structural profiling takes as input an antibody/BCR repertoire snapshot containing heavy (VH) and light (VL) chain reads. It eliminates VH and VL chains for which not every CDR is modellable. All modellable VH and VL chains are then sequence clustered to reduce computational complexity. Surviving cluster centres are then paired together and the resulting Fvs that are likely to be successfully modelled are retained. Finally, predicted modellable Fvs with the same combinations of CDR lengths are structurally clustered based on the orientation and CDR loop templates forecast to be used during homology modelling. Antibody Model Libraries ('AMLs') can then be built from these representative $\mathrm{Fv}$ sequences.

Structurally Profiling the Baseline Immune Repertoire. We first investigated the structural diversity present in the 41 selected Gidoni baseline repertoire datasets. Separately, each dataset was fed through our structural profiling pipeline to identify the set of sequence diverse modellable VH and VL domains, then the number of predicted modellable Fvs, and finally the number of distinct structures in each dataset (Table 1, full table available as SI Table 2).

The most structurally diverse dataset was 'S64' (209,394 dis-

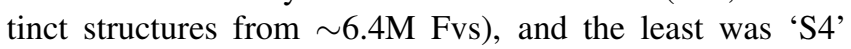
(78,588 distinct structures, from $\sim 750 \mathrm{~K}$ Fvs). Datasets with a larger number of modellable sequence diverse VHs tended to result in a larger number of distinct structures. Datasets with a moderate/low number of modellable sequence diverse VHs but very large numbers of modellable sequence diverse VLs were amongst the least structurally diverse (e.g. 'S95'). This is consistent with our understanding of both length and 
Table 1. Structurally profiling the baseline repertoire snapshots (30). A full table containing the values for all 41 baseline datasets is available in the Supporting Information (SI Table 2). In order, the columns show: the dataset label, the number of VH and VL reads within each snapshot, the number of FREAD-modellable VH and VL reads (once clustered at $90 \%$ sequence identity), the number of predicted modellable Fvs resulting from these $\mathrm{VH}$ - $\mathrm{VL}$ pairings, and the number of distinct structures (cluster centres) identified in each dataset. SIC $=$ Sequence Identity Clustered.

\begin{tabular}{|l|l|l|l|l|l|l|}
\hline Dataset & All VH & All VL & $\begin{array}{l}\text { Modellable VH } \\
{[90 \% \text { SIC }]}\end{array}$ & $\begin{array}{l}\text { Modellable VL } \\
{[90 \% \text { SIC] }}\end{array}$ & $\begin{array}{l}\text { Predicted } \\
\text { Modellable Fvs }\end{array}$ & Distinct Structures \\
\hline 1 (S64) & 177,603 & 123,934 & 10,087 & 6,779 & $6,420,211$ & 209,394 \\
2 (S57) & 169,805 & 118,020 & 9,860 & 7,922 & $7,225,630$ & 201,039 \\
3 (S5) & 159,544 & 139,845 & 8,999 & 8,526 & $6,827,419$ & 200,708 \\
4 (S56) & 162,446 & 136,874 & 9,309 & 7,168 & $6,628,683$ & 195,061 \\
5 (S83) & 152,299 & 112,733 & 9,048 & 8,076 & $6,170,373$ & 193,384 \\
6 (S67) & 173,722 & 120,237 & 9,349 & 6,424 & $5,544,952$ & 193,061 \\
7 (S84) & 164,017 & 138,874 & 8,702 & 8,232 & $5,634,598$ & 191,617 \\
8 (S76) & 148,180 & 126,713 & 8,778 & 7,047 & $5,856,150$ & 191,162 \\
9 (S54) & 121,993 & 133,921 & 7,581 & 9,066 & $5,074,822$ & 181,290 \\
10 (S89) & 152,710 & 144,340 & 8,923 & 9,293 & $5,414,820$ & 177,829 \\
$\ldots$ & $\ldots$ & $\ldots$ & $\ldots$ & $\ldots$ & $\ldots$ \\
39 (S95) & 118,576 & 162,377 & 5,412 & 11,748 & $5,901,443$ & 91,855 \\
40 (S17) & 102,405 & 111,669 & 5,310 & 7,945 & $2,690,081$ & 91,229 \\
41 (S4) & 100,689 & 128,986 & 4,688 & 1,761 & 745,977 & 78,588 \\
\hline
\end{tabular}

structural variability in $\mathrm{VH}$ (particularly in CDRH3) relative to VL (31-33).

\section{Expected Numbers of Distinct Structures (via. 'Ran-} dom Repertoires'). To contextualise the numbers of distinct structures observed for each baseline repertoire, we generated 'Random Repertoires' to obtain expected numbers of distinct structures assuming each genuine repertoire sampled randomly from modellable, accessible structure space. To achieve this, we derived:

(a) The Modellable Repertoire Structures: a sample of over 180 million structures built from a random combination of any orientation template, a CDR3 template, and a pair of CDR1/CDR2 templates from the same SAbDab entry (mimicking $\mathrm{V}$ gene-encoded predetermination).

(b) The Length-Accessible Repertoire Structures for each baseline snapshot: the subset of the Modellable Repertoire Structures with a CDR length combination observed in that individual.

(c) A 'Random Repertoire' for each baseline snapshot: the appropriate Length-Accessible Repertoire Structures dataset was sampled the same number of times as that individual's number of predicted modellable Fvs. Clustering these RRs then provided a reference number for the expected number of distinct structures per repertoire, given the depth of sampling in each dataset and assuming random sampling.

To derive a set of Modellable Repertoire Structures, we took the same number of samples as the number of Fvs derived from all baseline repertoire snapshots $(183,544,740$, SI Table 2). Upon structurally clustering, these samples yielded $\sim 28.7 \mathrm{M}$ distinct structures over $\sim 61.2 \mathrm{~K}$ distinct combinations of CDR lengths, roughly 100x as many distinct structures as seen in any baseline repertoire sample. However, as each repertoire snapshot typically only contained between 2,000-3,500 different CDR length combinations, many of these $28.7 \mathrm{M}$ distinct structures would never be observed in the real data. Therefore, 41 'Length-Accessible Repertoire
Structures' datasets were created, limiting the Modellable Repertoire Structures to the CDR length combinations seen in each snapshot. For example, considering only the 3,468 CDR length combinations observed in our most structurally diverse individual ('S64') reduced the Modellable Repertoire Structures to a Length-Accessible Repertoire Strucutres dataset of $\sim 154.8 \mathrm{M}$ structures. This clustered into $\sim 20.8 \mathrm{M}$ distinct structures (a 27.5\% reduction from the Modellable Repertoire Structures, while the number of CDR length combinations dropped $\sim 94 \%$ ), implying we have good structural sampling over the CDR length combinations typically seen in humans. Every Length-Accessible Repertoire Structures dataset contained a number of randomly-selected structures roughly 20-30 times larger than the number of predicted modellable Fvs observed in the corresponding baseline repertoire.

Finally, 41 separate 'Random Repertoires' were created to determine the expected number of distinct structures assuming random structural sampling and given the observed structural sampling depth (see Methods). To do this, each individual's Length-Accessible Repertoire Structures were sampled randomly, without replacement, the same number of times as the number of predicted modellable Fvs (Table 2).

Again taking 'S64' as an example, the 6,420,211 samples comprising 'Random Repertoire S64' yielded 2,187,257 distinct structures, equating to an average of 2.94 Fvs per distinct structure, compared to 30.66 (10.45x more) Fvs per distinct structure in the genuine repertoire. This provides strong evidence that the modellable portions of antibody repertoires occupy a highly focused region of modellable structure space - roughly $10 \%$ of the expected number given the sample size (Fig. 1), and $1 \%$ of a theoretical maximum estimate, across the same CDR length combinations.

'Public Baseline' Structures in Unrelated Individuals. We next investigated whether structural commonality exists between baseline repertoire snapshots. This phenomenon 
bioRxiv preprint doi: https://doi.org/10.1101/2020.03.17.993444; this version posted April 21, 2020. The copyright holder for this preprint (which was not certified by peer review) is the author/funder, who has granted bioRxiv a license to display the preprint in perpetuity. It is made available under aCC-BY-ND 4.0 International license.
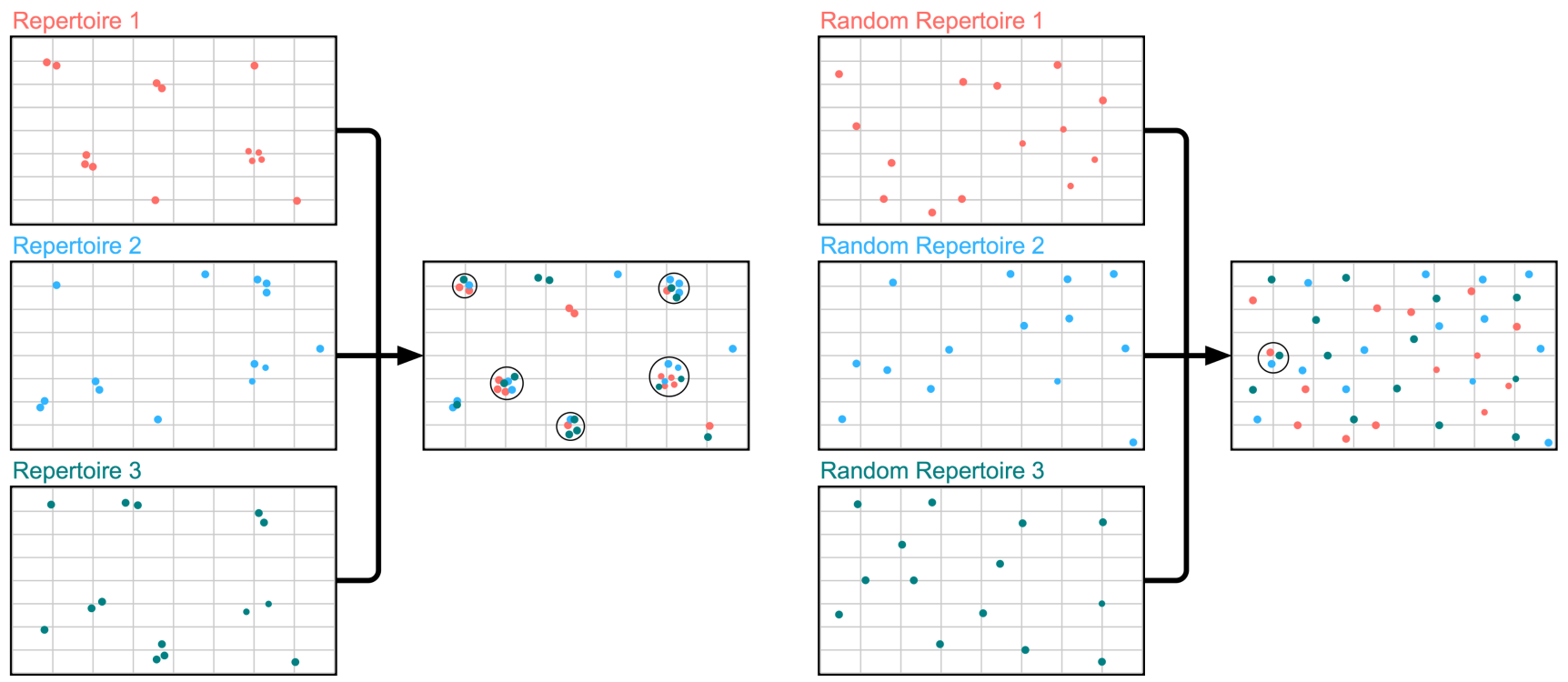

Fig. 1. Comparing genuine repertoire snapshot to synthetic 'Random Repertoires' (RRs). Each dot represents a distinct structure mapped onto a two-dimensional representation of 'Length-Accessible Repertoire Structure' space. The genuine repertoire snapshots of all three individuals (red $=$ repertoire 1, blue $=$ repertoire 2 , green $=$ repertoire 3) exhibit focused structural sampling, covering $\sim 10 \%$ of the space as the corresponding RRs. Overlap analysis shows a high proportion of genuine repertoire distinct structures can characterise an Fv in all three individuals ('public structures', represented by black circles). When the same overlap analysis is performed on the equivalent 'Random Repertoires', far fewer public structures are observed.

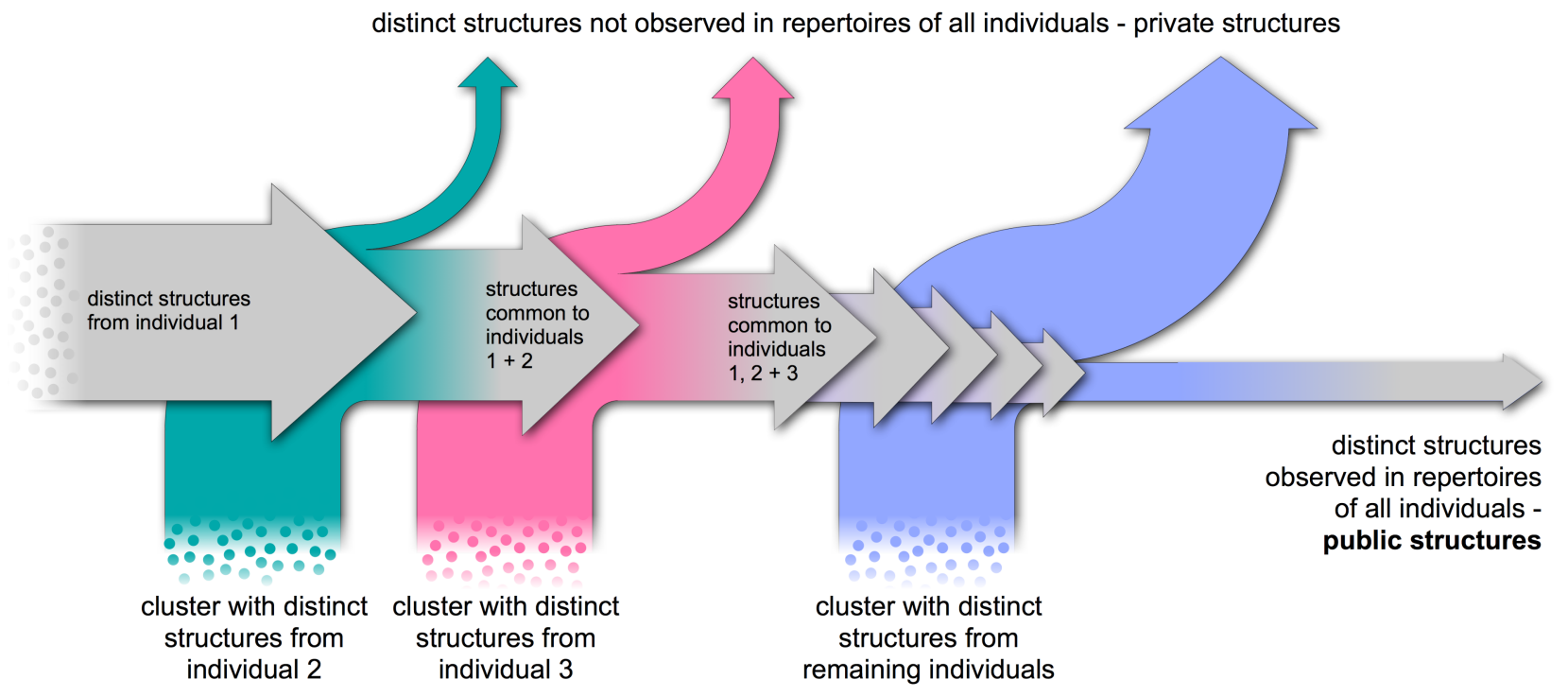

Fig. 2. Structural overlap analysis. Datasets are arranged in order of their internal structural diversity (most diverse first). Distinct baseline structures from individual 1 are clustered sequentially with all other repertoire snapshots. Distinct structures present in every tested dataset are classed as 'public structures', whereas those that are absent in at least one individual are termed 'private structures'.

would be statistically extremely unlikely by chance, given the focused structural sampling observed in each repertoire. To do this, we performed structural clustering on pairs of repertoire snapshots, looking for evidence of structural overlap (i.e. distinct structures assigned to a predicted modellable Fv seen in both datasets, see Methods and Fig. 2).

Repertoire snapshots were ordered by their internal structural diversity ('S64' first, through to 'S4'). The 209,394 distinct structures of S64 act as a reference set of cluster centres. The 7,225,630 Fvs from snapshot S57 were then compared to these S64 cluster centres. Structures present in both S57 and S64 were termed public across two individuals, while S64 and S57 distinct structures unique to their own dataset were termed private. Next, the 6,827,419 Fvs from S5 were compared to all public and private distinct structures observed in S64 and S57. We again evaluated the number of public structures, this time present in all three datasets. We repeated this analysis for all remaining baseline repertoire snapshots (first ten results in Table 2, all 41 results in SI Table 3).

To date, all in silico analysis of antibody repertoires has sug- 
Table 2. Public structure analysis across the ten most structurally diverse baseline repertoire snapshots. A table tracking the public structures across all datasets is available as SI Table 3. A statistical estimate for the number of public structures was derived by randomly sub-sampling each Random Repertoire to the yield the same number of distinct structures as its equivalent baseline repertoire snapshot. The 'Public Baseline' Antibody Model Library was derived from the 27,389 shared structures up to volunteer S89.

\begin{tabular}{|l|l|l|l|l|}
\hline $\begin{array}{l}\text { \# of Repertoires } \\
\text { (Dataset Added) }\end{array}$ & $\begin{array}{l}\text { Predicted Modellable } \\
\text { Fvs Added }\end{array}$ & $\begin{array}{l}\text { Cumulative } \\
\text { Public \& Private } \\
\text { Distinct Structures }\end{array}$ & $\begin{array}{l}\text { Public Distinct Structures } \\
\text { (Overall \% Public) }\end{array}$ & $\begin{array}{l}\text { Expected Public } \\
\text { Distinct Structures } \\
\text { (Overall \% Public) }\end{array}$ \\
\hline $1($ S64) & $6,420,211$ & 209,394 & 209,394 & 209,394 \\
$2(+$ S57) & $7,225,630$ & 340,915 & $100,824(29.57 \%)$ & $10,455(3.07 \%)$ \\
$3(+$ S5) & $6,827,419$ & 445,045 & $71,743(16.12 \%)$ & $1,351(0.30 \%)$ \\
$4(+$ S56) & $6,628,683$ & 527,668 & $58,043(11.00 \%)$ & $293(0.06 \%)$ \\
$5(+$ S83) & $6,170,373$ & 604,124 & $48,703(8.06 \%)$ & $78(0.01 \%)$ \\
$6(+$ S67) & $5,544,952$ & 670,833 & $42,277(6.30 \%)$ & $32(<0.01 \%)$ \\
$7(+$ S84) & $5,624,598$ & 734,374 & $37,151(5.06 \%)$ & $16(<0.01 \%)$ \\
$8(+$ S76) & $5,856,150$ & 793,831 & $30,474(3.60 \%)$ & $9(<0.01 \%)$ \\
$9(+$ S54) & $5,074,822$ & 846,670 & $27,389(3.06 \%)$ & $5(<0.01 \%)$ \\
$10(+$ S89) & $5,414,820$ & 896,328 & & $4(<0.01 \%)$ \\
\hline
\end{tabular}

gested that this number should drop rapidly towards 0 . For example, a recent clonotype analysis of the baseline circulating repertoire estimated that only around $0.022 \%$ of clonotypes were public across ten unrelated individuals (30). However, using our methodology, we found that the number of public distinct structures decreased at a far slower rate, still totalling 27,389 structures after ten unrelated individuals (Table 2). This represents $3.06 \%$ of all distinct structures observed up to that point, over 100 times the number of public clonotypes found by Briney et al. in their much deeper repertoire samples. Clonotyping our baseline snapshots by the same method, we observed just 26 public clonotypes across the first two repertoires (S64 and S57), and no public clonotypes across the first three repertoires (S64, S57, and S5).

To provide a statistical estimate for how many distinct structures would be expected to be shared across these ten baseline repertoires, the Random Repertoire distinct structures were subsampled to match the corresponding number of baseline repertoire distinct structures (see Methods). In contrast to the genuine repertoires, the Random Repertoires overlapped sparsely, reaching single digits of public structures by just the seventh volunteer (Table 2).

We also tracked the cumulative number of public and private structures over all 41 baseline repertoire snapshots (SI Table 4). Even after the first few most diverse datasets, the deviation from an expected number of distinct structures (given the same ratio of distinct structures:modellable Fvs observed in S64) is quite substantial. This suggests that we might not expect much deviation from our observed fraction of public baseline distinct structures upon deeper repertoire sampling. The existence of such a large number of 'Public Baseline' structures would be statistically extremely unlikely without the presence of underlying functional commonality. Clonotyping is fundamentally unable to capture the same depth of signal, even on much deeper sequencing samples, as functional selection is occuring at the level of structural and paratopic similarity, which may not correspond with conservation of gene transcript origin or high CDRH3 sequence identity. Our structural profiling approach is therefore the first computational method to provide supporting evidence for the levels of baseline functional equivalence long hypothesised by immunologists.

The 'Public Baseline' Antibody Model Library. Predicted structures shared between many individuals might represent good starting points for therapeutic development. Their widespread nature could point to their binding versatility, and also to broad immune system tolerance across many individuals, lowering the risk of drug immunogenicity.

We used ABodyBuilder (27) to construct an Antibody Model Library (AML) based on the 27,389 'S64' pairings predicted to adopt a 'Public Baseline' structure (as defined by the ten most structurally diverse repertoire snapshots). Some Fvs failed to be entirely homology modelled. For example, occasionally the CDRH3 template clashes irreparably with the CDRL3 template during construction of the full $\mathrm{Fv}$ model, necessitating ab initio treatment. Overall, 23,700 (86.53\%) of 27,389 pairings were entirely homology modelled and comprise our 'Public Baseline AML' (SI Dataset 1).

We then investigated the reported species origin of the templates used to model the loops within our 'Public Baseline AML' (Table 3). While this is only an approximate measure, as most PDB antibody structures are engineered to some extent, human-origin templates were used considerably more often to model the 'Public Baseline' Fvs than would be expected from their abundance in the modelling database

Table 3. The percentage of each Complementarity-Determining Region's (CDR's) templates in the database with reported human origin, against the percentage usage of human-derived templates for CDRs in the 'Public Baseline' Antibody Model Library (AML).

\begin{tabular}{|l|l|l|}
\hline CDR & $\begin{array}{l}\text { Human Templates, } \\
\text { Database (\%) }\end{array}$ & $\begin{array}{l}\text { Human Templates, } \\
\text { Public Baseline AML } \\
(\%)\end{array}$ \\
\hline H1 & 37.99 & 93.46 \\
H2 & 37.85 & 89.08 \\
H3 & 36.47 & 72.63 \\
L1 & 46.85 & 95.69 \\
L2 & 47.00 & 92.56 \\
L3 & 47.07 & 89.51 \\
\hline
\end{tabular}


(a)

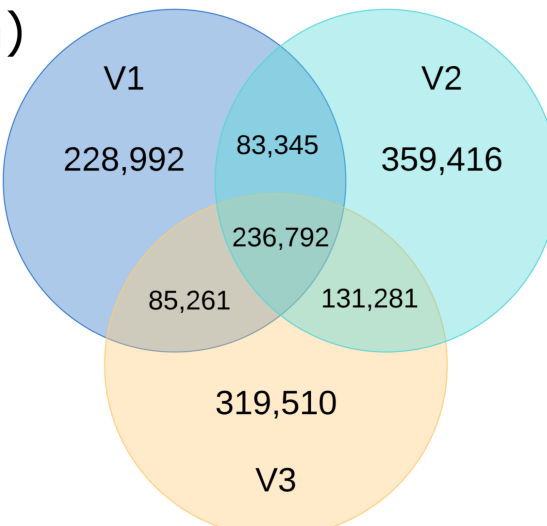

Before Vaccination (BV) (b)

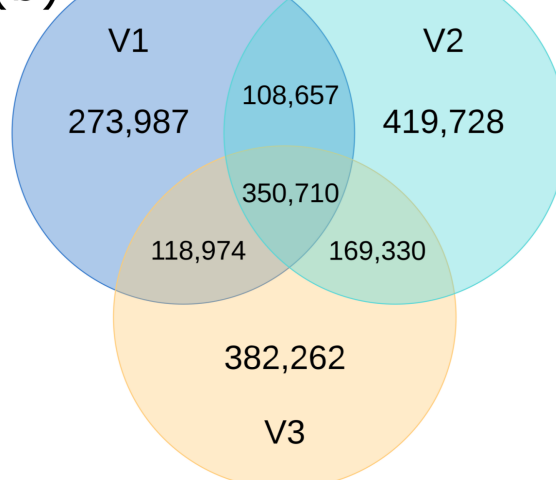

After Vaccination (AV)

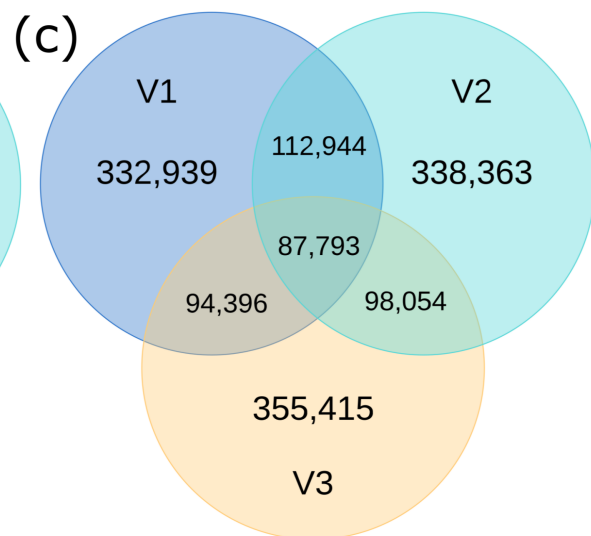

Pure After Vaccination (PAV)

Fig. 3. Venn diagrams showing the structural overlap between each individual's 'Before Vaccination' dataset (a), 'After Vaccination' dataset (b), and 'Pure After Vaccination' dataset ((c), distinct structures arising only after vaccination). Total distinct structures: Before Vaccination - 1,444,597; After Vaccination - 1,823,628; Pure After Vaccination $1,419,904$. V1-V3 = Volunteer $1-3$

(36.47\% - 47.07\% abundance; $72.63 \%-95.69 \%$ usage). This further emphasises the value added by starting with human antibody repertoire data, and could indicate that our 'Public Baseline AML' structures possess a lower risk of intrinsic immunogenicity to humans.

To test whether our 'Public Baseline AML' already contains antibodies proximal to known therapeutics, we mined TheraSAbDab (34) for all $100 \%$ sequence identical structures of WHO-recognised therapeutics, selecting one per therapeutic (see Methods). Of the 66 therapeutics with known structures that had at least one antibody in our 'Public Baseline AML' with identical CDR lengths, all had a structural partner in the AML within a $\mathrm{C}_{\alpha}$ Fv RMSD of $1.84 \AA$, and $37(56.1 \%)$ had a structural partner within $1.00 \AA$ Fv RMSD. Eleven therapeutic structures lay within $0.75 \AA$ Fv RMSD of a 'Public Baseline AML' structure (SI Table 5); these therapeutics spanned a wide range of targets and were primarily successful or promising drugs (4 approved, 5 active in Phase III, 1 active in Phase II, and 2 discontinued).

This result demonstrates that the antibody models within our 'Public Baseline AML', without any explicit design, can display high levels of geometric similarity to known therapeutics. Screening libraries based on these commonly shared structures holds significant promise for finding novel lowimmunogenicity therapeutics.

Structurally Profiling a Flu Vaccine Response. Clonotyping is commonly used in antibody drug discovery to identify 'expanded clones' - novel genetic lineages present after vaccination/infection but that were absent, or low concentration, beforehand (14). Here, we applied our repertoire characterisation method to investigate whether we could identify an analogous structural response to vaccination.

To this end, we used a longitudinal 2009 seasonal flu vaccination study by Gupta et al. (5), in which three unrelated individuals ('V1-3') were sequenced at many time-points before and after vaccination. Sequences were again downloaded from the OAS database, yielding 'Before Vaccination' and 'After Vaccination' datasets for each individual, according to the protocol described in the Methods. Using the same repertoire structural profiling protocol as above, we calculated the number of distinct structures observed in each individual before and after vaccination (SI Table 4).

To obtain an estimate for the degree of structural commonality pre- and post-vaccination, we again used a greedy clustering approach to evaluate the structural overlap between the 'Before Vaccination' datasets, and between the 'After Vaccination' datasets, separately (Fig. 3a, 3b). The first dataset in each overlap assessment was the most structurally diverse (i.e. the ' $\mathrm{V} 3$ ' individual before vaccination, and ' $\mathrm{V} 1$ ' after vaccination).

Again, a significant number of public distinct structures were observed in 'V1', 'V2', and 'V3' ('Public Before Vaccination' structures, $17.78 \%(236,792 / 1,444,597)$ of all 'Before Vaccination' distinct structures). This indicates that the identification of 'Public Baseline' structures in the previous section was unlikely due to serendipitous Ig-seq amplification bias. Interestingly, $17.78 \%$ is a similar percentage of sharing as that seen after three baseline snapshots $(16.12 \%$; $71,743 / 445,045)$.

The degree of structural sharing appears to increase after vaccination, with $19.23 \%(350,710 / 1,823,648)$ public structures across the three volunteers. This is consistent with a degree of repertoire structural convergence driven by exposure to the same pathogenic epitopes.

To derive these convergent structures, the structural overlap between each individual's 'Before Vaccination' and 'After Vaccination' datasets was measured, only retaining 'After Vaccination' pairings that could not be clustered into the same individual's 'Before Vaccination' distinct structures. ' $V 1$ ' remained the most structurally diverse dataset, with 628,072 'Pure After Vaccination' distinct structures. The 
overlap between these 'Pure After Vaccination' pairings (Fig. $3 \mathrm{c})$ was then compared. This yielded a mixed picture of convergent and private vaccination response structures - $27.7 \%$ $(393,187 / 1,419,904)$ of distinct structures were shared with at least one other individual, and $6.18 \%(87,793 / 1,419,904)$ were shared across all three individuals - which we term 'Public Response' structures.

There are two potential causes of overlap in the 'Pure After' vaccination set. One is a genuine common structural response to vaccination, while the other is that the initial baseline repertoire was under-sampled - i.e. the overlap reflects residual shared baseline structures. As a second test for baseline deviation, beyond absence before vaccination, we compared how many of the 27,389 'Public Baseline' distinct structures were within $1 \AA$ of a 'Public Before Vaccination' binding site, versus the number within $1 \AA$ of a 'Public Response' Structure binding site. We observed that $80.0 \%(21,922 / 27,389)$ of 'Public Baseline' structures were within $1 \AA$ of a 'Public Before Vaccination' structure, compared to just $24.2 \%$ $(6,621 / 27,389)$ proximal to a 'Public Response' structure. This provides further evidence that a proportion of these convergent 'Public Response' structures reside in a distinct region of structural space and could harbour epitope-specific binding geometries. We have built a 'Public Response AML' based on these 87,793 shared structures, with 74,181 Fvs (84.4\%) successfully homology modelled (SI Dataset 2).

\section{Discussion}

In this work, we have structurally profiled antibody repertoires to capture new insights into the baseline and antigenresponding immune system, and to create novel libraries of antibody model structures that could be exploited for immunotherapeutic discovery.

All of the structural analysis in this paper is limited to the antibody chains that are currently predicted to be modellable, and so there remain regions of natural structural space uninvestigated. Nevertheless, we show that antibody repertoires tend only to explore highly focused regions of currentlymodellable structural space $(\sim 10 \%$ of the diversity expected if templates were explored randomly across the same combinations of CDR lengths). This suggests that the current degree of structural commonality would remain across the (as yet) unmodellable regions of structural space.

The enormous sequence diversity exhibited across baseline antibody repertoires has long appeared to run contrary to the theory of baseline functional commonality. Here we have shown that, at least from a structural perspective, there is considerable opportunity for functional commonality across the circulating resting-state repertoires of unrelated individuals ( $\sim 3 \%$ of observed distinct structures are public across 10 individuals). The theoretical chemical diversity that could be displayed on each of these scaffolds is large, so many of these grouped binding sites will not be complementary to the same antigen epitope. However, there is good reason to believe that a certain proportion are, as geometric similarity is a likely prerequisite of functional commonality, and our structural clustering approach offers a route to detecting and analysing these antibodies. This knowledge could then be harnessed in vaccinology - for example, identifying an epitope targetable by a 'Public Baseline' structure may lead to a more reliable and convergent response.

We hypothesise that human 'Public Baseline' structures are more likely to display low levels of human immunogenicity and be versatile binders. Building full three-dimensional variable domain models of these distinct structures (an Antibody Model Library) produced geometries that were very close to several approved and late-stage active therapeutic antibodies targeting diverse antigens. To chemically elaborate this 'Public Baseline' structural basis set, a phage display library on the order of $10^{6}-10^{7}$ sequence-unique human antibodies could be created from the many different Fv sequences predicted to adopt each public distinct structure.

Target-focused screening libraries against immunodominant epitopes are commonly derived through sequence analysis of longitudinal Ig-seq studies that track the immune response of many individuals to the same antigen. We show that when our methodology is applied to a longitudinal flu vaccination case study, we detect a higher level of structural convergence, commensurate with response to similar epitopes on the same antigen. We can also derive a large number of 'Public Response' structures, with divergent structural characteristics from the baseline repertoire. These could contain useful binding site structures exploitable for antigen-specific library design.

There are inevitable biases in structurally profiling human antibody repertoire data to suggest antibody leads for drug discovery. One such biased property is CDRH3 length: very short CDRH3 lengths will be under-sampled through their sparsity in natural human sequences (29), while very long CDRH3 lengths will be under-sampled because they are more difficult to homology model accurately. While inherent immunogenicity should be diminished by virtue of using naturally-expressed sequences, other developability issues are still possible, as not every human antibody has the biophysical properties ideal for large-scale manufacture and long-term storage (29).

Nevertheless, we believe that our approach should find immediate applicability in both in silico and in vitro screening. We have made available the 'Public Baseline' and 'Public Response' Antibody Model Libraries for further investigation, and will continue to build and share the Antibody Model Libraries derived from other unpaired and paired $\mathrm{VH}+\mathrm{VL}$ datasets in the Observed Antibody Space database (9).

\section{Methods}

Immunoglobulin Gene Sequencing Datasets. The cleaned and translated antibody repertoire datasets $(5,30)$ were downloaded directly from the Observed Antibody Space (OAS) database (9). For the Gidoni data (30), only individuals for whom $>100,000 \mathrm{IgM} \mathrm{VH}$ and $>100,000 \mathrm{VL}$ sequences were recorded were analysed. In our analysis of Gupta et al. (5), we used all three individuals ('V1' = 'FV', 'V2' = 'GMC', and 'V3' = 'IB'). The 'Before Vaccination' data was defined as all $\mathrm{VH}$ and $\mathrm{VL}$ sequences recorded at 
8 days, 2 days and 1 hour before vaccination. The 'After Vaccination' data was defined as all VH and VL sequences recorded at 1 week, 2 weeks, 3 weeks, and 4 weeks after vaccination. Sequences recorded 1 hour and 1 day after vaccination were discarded to avoid ambiguity. The 'Pure After Vaccination' data contained 'After Vaccination' sequences that did not fall into the structural clusters defined by each individual's 'Before Vaccination' repertoires. The seminal work in which 'FV', 'GMC', and 'IB' were vaccinated is detailed in Laserson et al. (4), however the data we use derives from Gupta et al. (5), who re-analysed each antibody repertoire snapshot with Illumina sequencing.

Repertoire Structural Profiling Pipeline. The described structural profiling pipeline was optimised from the protocol reported in the Supporting Information of Proc. Natl. Acad. Sci. (2019) 110(6):4025-4030 (29).

CDR modellability analysis. Each sequence was first numbered using ANARCI (35) according to the IMGT numbering scheme (36), and the closest framework region (variable domain with North-defined CDRs (31) excised) in the SAbDab (23) database $\left(12^{\text {th }}\right.$ February 2019$)$ was identified. In the IMGT numbering scheme, the North CDRs lie between the following residue numbers - CDRH1: 24-40; CDRH2: 5566; CDRH3: 105-117; CDRL1: 24-40; CDRL2: 55-69; CDRL3: 105-117.

FREAD $(37,38)$ was then used to attempt to map each Igseq sequence to a length-matched North CDR template. The FREAD CDR databases were timestamped to $12^{\text {th }}$ February 2019, and contained the following numbers of templates CDRH1: 2,526; CDRH2: 2,575; CDRH3: 2,502; CDRL1: 2,355; CDRL2: 2,373; CDRL3: 2,376. All loop templates contain the North-defined CDR loop and 5 'anchor residues' before and after the loop. Selection of CDRH3 templates was performed according to a bespoke set of EnvironmentSpecific Substitution Score (ESS) thresholds established using Ig-seq data: Lengths 5-8, ESS $\geq 25$; Lengths 9-10, ESS $\geq 35$; Lengths $11+$, ESS $\geq 40$ (see SI Methods). Each template surpassing the threshold was subsequently grafted onto the corresponding framework anchor residues. The loop template with the lowest calculated $\mathrm{C}_{\alpha}$ anchor RMSD was selected. Any sequences for which at least one loop could not be modelled were removed from the dataset.

Sequence clustering. The modellable chains were then sequence clustered using CD-HIT (39) at a 90\% sequence identity threshold, to reduce the number of $\mathrm{VH}-\mathrm{VL}$ pairing comparisons to a computationally-tractable number.

Predicting modellable VH-VL orientations. The 20 most important VH-VL interface residues for orientation prediction were derived; a sequence identity of $85 \%$ over these 20 residues resulted in an orientation RMSD of $\leq 1.5 \AA \sim 80 \%$ of the time (see SI Methods).

All remaining $\mathrm{VH}$ and $\mathrm{VL}$ domains after sequence clustering were paired together, and their 20 key interface residues were recorded. If the sequence identity over these residues was $\geq$
$85 \%$ to at least one of 1,129 reference Fvs, the interface was classed as modellable - otherwise the VH-VL pairing was discarded. If multiple reference Fvs shared $\geq 85 \%$ identity, the predicted modellable $\mathrm{VH}-\mathrm{VL}$ pairing inherited the orientation parameters of the Fv reference with highest sequence identity.

Identifying distinct structures. At this stage, each predicted modellable VH-VL pairing (Fv) has eight associated parameters: its orientation template, its six CDR templates, and a length vector recording the combination of North CDR lengths (31) present in its binding site. Fvs were then structurally clustered to identify 'distinct structures' according to the following process. First, identically-predicted binding sites (for which the eight predicted parameters were the same) were identified. The retained pairing was randomly chosen, except in the overlap studies - if one of the pairings was present as a distinct structure of the first dataset, this pairing was selected and recorded as a shared structure across both repertoires.

Next, singleton length clusters were identified and assigned as separate distinct structures, avoiding inaccurate RMSD comparisons between loops of differing length. The remaining interfaces were split by their CDR length combinations, and were greedily clustered with all other pairings sharing the same length vector as follows:

1. Select the first pairing as a distinct structure (cluster centre).

2. Select the next pairing. If the orientation RMSD to all existing cluster centre orientation templates exceeds $1.5 \AA$, classify the new pairing as a distinct structure. Otherwise:

3. Calculate the RMSD between the CDR templates of the new pairing with those of all existing cluster centres using the formula:

$$
\sqrt{\frac{\sum_{X}^{(H 1-H 3, L 1-L 3)} D_{X_{12}}^{2} L_{\mathrm{X}}}{\sum_{X}^{(H 1-H 3, L 1-L 3)} L_{\mathrm{X}}}}
$$

where the sum over $\mathrm{X}$ refers to each of the six CDRs, $\mathrm{L}_{\mathrm{X}}$ is the length of North CDRX, and $\mathrm{D}_{X_{12}}$ is the $\mathrm{C}_{\alpha}$ RMSD between the CDRX in Fv 1 and $F v 2$. If this value exceeds $1 \AA$ to all existing structural cluster centres, the pairing is assigned as a distinct structure. Otherwise the pairing is stripped from the dataset.

4. Return to step 2 until all pairings have been analysed.

Overlap Comparison. To identify shared structures between two Ig-seq repertoire snapshots, the distinct structures from the first snapshot were listed followed by all predicted modellable Fvs of the second repertoire snapshot, as an input file to the clustering algorithm. The greedy clustering ensured that all distinct structures from the first dataset remained as cluster centres, and allowed for the identification of pairings in the second dataset that were also predicted to occupy the same structural neighbourhood. 
bioRxiv preprint doi: https://doi.org/10.1101/2020.03.17.993444; this version posted April 21, 2020. The copyright holder for this preprint (which was not certified by peer review) is the author/funder, who has granted bioRxiv a license to display the preprint in perpetuity. It is made available under aCC-BY-ND 4.0 International license.

'Random Repertoires'. To contextualise the structural diversity displayed in human antibody repertoires, we derived 'Random Repertoires' (RRs) according to the following method. First, a set of Modellable Repertoire Structures (MRS) was generated. When generating a structure, one of any of the 1,129 orientation templates, 2,502 FREAD CDRH3 templates, and 2,376 FREAD CDRL3 templates were available for selection. To mirror the genetics of the immune system (as they would be encoded on the same $\mathrm{V}$ gene transcript), CDR1 and CDR2 templates were restricted to being selected from the same SAbDab structure, limiting our choice to one of 2,519 CDRH1/2 templates and 2,345 CDRL1/2 templates. Each of these five sets was randomly sampled over 180 million times to create the MRS dataset. This was then filtered to create 41 Length-Accessible Repertoire Structure (LARS) datasets, containing only the combinations of CDR lengths observed in each baseline repertoire snapshot. Finally, RRs were created by sampling each LARS set the same number of times as the number of predicted modellable Fvs in the corresponding baseline repertoire snapshot.

To obtain statistically expected values for structural overlap across individuals, the distinct structures from 'RR_S64' were randomly subsampled the same number of times as the number of distinct structures seen in 'S64' itself, yielding random distinct structure samples occupying the same proportion of LARS-space. The process was repeated for each $\mathrm{RR}$ dataset, normalising to each respective baseline repertoire snapshot. Overlap comparison was then performed as described above, starting from the 'RR_S64' distinct structures, followed by all the pairings that fell into the selected 'RR_S57' distinct structures, etc.

Clonotyping. Clonotyping was performed to group antibodies with the same closest $\mathrm{V}$ and $\mathrm{J}$ gene, and identical CDRH3 sequences, as in Briney et. al. (1).

Antibody Model Library Construction. Antibody model libraries (AMLs) were constructed with a parallel implementation of ABodyBuilder (27), using the FREAD $(37,38)$ Environment Specific Substitution Scores derived from Ig-seq benchmarking (see CDR Modellability Analysis). Some predicted modellable Fvs are not entirely homology modellable, as loop modellability is considered on a per-chain basis and does not take into account inter-chain loop clashes that become evident only upon full Fv homology modeling. Fvs that required any degree of ab initio modelling to fix such issues were trimmed out of the dataset.

Comparison to Antibody Therapeutics. The set of 89 therapeutics with 100\% sequence identical structures (as of November 2019) were retrieved from Thera-SAbDab (34). A single structure was chosen for each therapeutic for the RMSD analysis; if multiple structures were available, we selected unbound structures with the best resolution. RMSD comparisons were only made between therapeutics and AML structures with matching combinations of CDR lengths. Fv
RMSD was calculated over all $\mathrm{C}_{\alpha}$ atoms after alignment of backbone atoms, using an in-house script.

\section{ACKNOWLEDGEMENTS}

This work was supported by an Engineering and Physical Sciences Research Council and Medical Research Council grant [EP/L016044/1] awarded to MIJR, a Biotechnology and Biological Sciences Research Council (BBSRC) grant [BB/M011224/1] awarded to AK, and funding from GlaxoSmithKline plc, UCB Pharma Ltd., AstraZeneca plc, and F. Hoffmann-La Roche AG.

\section{AUTHOR CONTRIBUTIONS}

M.I.J.R., C.M., and C.M.D. designed research; M.I.J.R. performed research; M.I.J.R., C.M., A.K., A.P.L., J.S., and C.M.D. analyzed data; and M.I.J.R., C.M., and C.M.D. wrote the paper.

\section{COMPETING FINANCIAL INTERESTS}

A.P.L. is employed by GlaxoSmithKline plc, and J.S. is employed by UCB Celltech. Both companies discover and sell antibody therapies.

\section{Bibliography}

1. Bryan Briney, Anne Inderbitzin, Collin Joyce, and Dennis R. Burton. Commonality despite exceptional diversity in the baseline antibody repertoire. Nature, 566:393-397, 2019. doi: 10.1038/s41586-019-0879-y.

2. Zhan Shi, Qingyang Zhang, Huige Yan, Ying Yang, Pingzhang Wang, Yixiao Zhang, Zhenling Deng, Meng Yu, Wenjing Zhou, Qianqian Wang, Xi Yang, Mo Xiaoning, Chi Zhang, Jing Huang, Hui Dai, Sun Baofa, Yongliang Zhao, Liang Zhang, Yun-Gui Yang, and Xiaoyan Qiu. More than one antibody of individual $B$ cells revealed by single-cell immune profiling. Cell Discov., 5:64, 2019. doi: 10.1038/s41421-019-0137-3.

3. Jacob D Galson, Johannes Trück, Anna Fowler, Elizabeth A Clutterbuck, Márton Münz, V Cerundolo, Claudia Reinhard, Robbert van der Most, Andrew J Pollard, Gerton Lunter, and Dominic F. Kelly. Analysis of B cell repertoire dynamics following hepatitis B vaccination in humans, and enrichment of vaccine-specific antibody sequences. EBioMedicine, 2(12): 2070-2079, 2015. doi: 10.1016/j.ebiom.2015.11.034.

4. Uri Laseron, Francois Vigneault, Daniel Gadala-Maria, Gur Yaari, Mohamed Uduman, Vander Heiden Jason A, William Kelton, Sang Taek Jung, Yi Liu, Jonathan Laserson, Raj Chari, Je-Hyuk Lee, Ido Bachelet, Brendan Hickey, Erez Lieberman-Aiden, Bozena Hanczaruk, Birgitte B Simen, Michael Egholm, Daphne Koller, George Georgiou, Steven H Kleinstein, and George M Church. High-resolution antibody dynamics of vaccine-induced immune responses. Proc. Natl. Acad. Sci. USA, 111(13):4928-4933, 2014. doi: 10.1073/pnas. 1323862111.

5. Namita T Gupta, Kristofor D Adams, Adrian W Briggs, Sonia C Timberlake, Francois Vigneault, and Steven $\mathrm{H}$ Kleinstein. Hierarchical clustering can identify B cell clones with high confidence in Ig repertoire sequencing data. J. Immunol., 198(6):2489-2499, 2017. doi: 10.4049/jimmunol.1601850.

6. Xueling Wu, Tongqing Zhou, Jiang Zhu, Baoshan Zhang, Ivelin Georgiev, Charlene Wang, Xuejun Chen, Nancy S Longo, Mark Louder, Krisha McKee, Sijy O'Dell, Stephen Perfetto, Stephen D Schmidt, Wei Shi, Lan Wu, Yongping Yang, Zhi-Yong Yang, Zhongjia Yang, Zhenhai Zhang, Mattia Bonsignori, John A Crump, Saidi H Kapiga, Noel E Sam, Barton F Haynes, Melissa Simek, Dennis R Burton, Wayne C Koff, Nicole A Doria-Rose, Mark Connors, NISC Comparative Sequencing Program, James C Mullikin, Gary J Nabel, Mario Roederer, Lawrence Shapirol, Peter D Kwong, and John R Mascola. Focused evolution of HIV-1 neutralizing antibodies revealed by structures and deep sequencing. Science, 333 (6049):1593-1602, 2011. doi: 10.1126/science.1207532.

7. Merle Schanz, Thomas Liechti, Osvaldo Zagordi, Enkelejda Miho, Sai T Reddy, Huldrych F Günthard, Alexandra Trkola, and Michael Huber. High-throughput sequencing of human immunoglobulin variable regions with subtype identification. PLoS One, 9(11):e111726, 2014. doi: 10.1371/journal.pone.0111726.

8. Jiang Zhu, Gilad Ofek, Yongping Yang, Baoshan Zhang, Mark K Louder, Gabriel Lu, Krisha McKee, Marie Pancera, Jeff Skinner, Zhenhai Zhang, Robert Parks, Joshua Eudailey, Krissey E Lloyd, Julie Blinn, S Munir Alam, Barton F Haynes, Melissa Simek, Dennis R Burton, Wayne C Koff, NISC Comparative Sequencing Program, James C Mullikin, John R Mascola, Lawrence Shapiro, and Peter D Kwong. Mining the antibodyome for HIV-1-neutralizing antibodies with next-generation sequencing and phylogenetic pairing of heavy/light chains. Proc. Natl. Acad. Sci. USA, 110(16):6470-6475, 2013. doi: 10.1073/pnas.1219320110.

9. Aleksandr Kovaltsuk, Jinwoo Leem, Sebastian Kelm, James Snowden, Charlotte M Deane, and Konrad Krawczyk. Observed Antibody Space: A resource for data mining nextgeneration sequencing of antibody repertoires. J. Immunol., 201(8):2502-2509, 2018. doi: 10.4049/jimmunol. 1800708 .

10. Victor Greiff, Ulrike Menzel, Enkelejda Miho, Cédric Weber, René Riedel, Skylar Cook, Atijeh Valai, Telma Lopes, Andreas Radbruch, Thomas H Winkler, and Sai T Reddy. Systems analysis reveals high genetic and antigen-driven predetermination of antibody repertoires throughout B cell development. Cell Rep., 19(7):1467-1478, 2017. doi: 10.1016/j.celrep.2017.04.054.

11. Brandon J DeKosky, Gregory C Ippolito, Ryan P Deschner, Jason J Lavinder, Yariv Wine, Brandon M Rawlings, Narin Varadarajan, Claudia Giesecke, Thomas Dörner, Sarah F Andrews, Patrick C Wilson, Scott P Hunicke-Smith, C Grant Wilson, Andrew D Ellington, and George Georgiou. High-throughput sequencing of the paired human immunoglobulin heavy and light chain repertoire. Nat. Biotechnol., 31:166-169, 2013. doi: 10.1038/nbt.2492.

12. Leonard D Goldstein, Ying-Jiun J Chen, Jia Wu, Subhra Chaudhuri, Yi-Chun Hsiao, Kellen Schneider, Kam Hon Hoi, Zhonghua Lin, Steve Guerrero, Bijay S Jaiswal, Jeremy Stinson, Aju Antony, Kanika Bajaj Pahuja, Dhaya Seshasayee, Zora Modrusan, Isidro Hötzel, and Somasekar Seshagiri. Massively parallel single-cell B-cell receptor sequencing enables rapid discovery of diverse antigen-reactive antibodies. Commun. Biol., 2:304, 2019. doi: 10.1038/s42003-019-0551-y. 
bioRxiv preprint doi: https://doi.org/10.1101/2020.03.17.993444; this version posted April 21, 2020. The copyright holder for this preprint (which was not certified by peer review) is the author/funder, who has granted bioRxiv a license to display the preprint in perpetuity. It is made available under aCC-BY-ND 4.0 International license.

13. Ian Setliff, Andrea R Shiakolas, Kelsey A Pilewski, Amyn A Murji, Rutendo E Mapengo, Katarzyna Janowska, Simone Richardson, Charissa Oosthuysen, Nagarajan Raju, Larance Ronsard, Masaru Kanekiyo, Juliana S Qin, Kevin J Kramer, Allison R Greenplate, Wyatt J McDonnell, Barney S Graham, Mark Connors, Daniel Lingwood, Priyamvada Acharya, Lynn Morris, and Ivelin S Georgiev. High-throughput mapping of B cell receptor sequences to antigen specificity. Cell, 179(7):1636-1646, 2019. doi: 10.1016/j.cell.2019.11.003.

14. Laura López-Santibáñez Jácome, S Eréndira Avendaño Vásquez, and Carlos Fabián Flores-Jasso. The pipeline repertoire for Ig-seq analysis. Front. Immunol., 10:899, 2019. doi: 10.3389/fimmu.2019.00899.

15. Uri Hershberg and Eline T Luning Prak. The analysis of clonal expansions in normal and autoimmune B cell repertoires. Philos. Trans. R. Soc. B Biol. Sci., 370:1676, 2015. doi: 10.1098/rstb.2014.0239.

16. Gur Yaari and Steven H Kleinstein. Practical guidelines for B-cell receptor repertoire sequencing analysis. Genome Med., 7:121, 2015. doi: doi.org/10.1186/s13073-015-0243-2.

17. Victor Greiff, Cédric R Weber, Johannes Palme, Ulrich Bodenhofer, Enkelejda Miho, Ulrike Menzel, and Sai T Reddy. Learning the high-dimensional immunogenomic features that predict public and private antibody repertoires. J. Immunol., 2017. doi: 10.4049/jimmunol. 1700594.

18. Anne Ljungars, Carolin Svensson, Anders Carlsson, Elin Birgersson, Ulla-Carin Tornberg, Björn Frendéus, Mats Ohlin, and Mikael Mattsson. Deep mining of complex antibody phage pools generated by cell panning enables discovery of rare antibodies binding new targets and epitopes. Front. Immunol., 10:847, 2019. doi: 10.3389/fphar.2019.00847.

19. Riu Yu, Shuang Wang, Yun-Zhou Yu, Wei-shi Du, Fang Yang, Wei-yuan Yu, and Zhi-wei Sun Neutralizing antibodies of botulinum neurotoxin serotype A screened from a fully synthetic human antibody phage display library. J. Biomed. Screen., 14(8):991-998, 2009. doi: 10. 1177/1087057109343206.

20. Karen Cerosaletti, Fariba Barahmand-pour Whitman, Junbao Yang, Hannah A DeBerg, Matthew J Dufort, Sara A Murray, Elisabeth Israelsson, Cate Speake, Vivian H Gersuk, James A Eddy, Helena Reijonen, Carla J Greenbaum, William W Kwok, Erik Wambre, Martin Prlic, Raphael Gottardo, Gerald T Nepom, and Peter S Linsley. Single-cell RNA-seq reveals expanded clones of islet antigen-reactive $\mathrm{CD} 4+\mathrm{T}$ cells in peripheral blood of subjects with type 1 diabetes. J. Immunol., 199(1):323-335, 2017. doi: 10.4049/jimmunol.1700172.

21. Erin M Mitsunaga and Michael P Snyder. Characterization of the human antibody response to natural infection using longitudinal immune repertoire sequencing. Mol. Cell. Proteom. 2019. doi: $10.1074 / \mathrm{mcp}$.RA119.001633.

22. Aleksandr Kovaltsuk, Konrad Krawczyk, Jacob D Galson, Dominic F Kelly, Charlotte M Deane, and Johannes Trück. How B-cell receptor repertoire sequencing can be enriched with structural antibody data. Front. Immunol., 8:1753, 2017. doi: 10.3389/fimmu.2017. 01753 .

23. James Dunbar, Konrad Krawczyk, Jinwoo Leem, Terry Baker, Angelika Fuchs, Guy Georges, Jiye Shi, and Charlotte M Deane. SAbDab: the Structural Antibody Database. Nucleic Acids Res., 42(D1):D1140-D1146, 2014. doi: 10.1093/nar/gkt1043.

24. Yibin Lin. What's happened over the last five years with high-throughput protein crystallization screening? Expert Opin. Drug Dis., 2018. doi: 10.1080/17460441.2018.1465924.

25. Konrad Krawczyk, Sebastian Kelm, Aleksandr Kovaltsuk, Jacob D Galson, Dominic Kelly, Johannes Trück, Cristian Regep, Jinwoo Leem, Wing K Wong, Jaroslaw Nowak, James Snowden, Michael Wright, Laura Starkie, Anthony Scott-Tucker, Jiye Shi, and Charlotte M Deane. Structurally mapping antibody repertoires. Front. Immunol., 9:1698, 2018. doi: 10.3389/fimmu.2018.01698.

26. Aleksandr Kovaltsuk, Matthew IJ Raybould, Wing Ki Wong, Claire Marks, James Snowden, Johannes Trück, and Charlotte M Deane. Structural diversity of B-cell receptor repertoires along the B-cell differentiation axis in humans and mice. PLoS Comp. Bio., 2020. doi: 10.1371/journal.pcbi.1007636.

27. Jinwoo Leem, James Dunbar, Guy Georges, Jiye Shi, and Charlotte M Deane. Abodybuilder: Automated antibody structure prediction with data-driven accuracy estimation. mAbs, 8(7):1259-1268, 2016. doi: 10.1080/19420862.2016.1205773.

28. Dimitri Schritt, Songling Li, John Rozewicki, Kazutaka Katoh, Kazuo Yamashita, Wayne Volkmuth, Guy Cavet, and Daron M Standley. Repertoire builder: high-throughput structural modeling of B and T cell receptors. Mol. Syst. Des. Eng., 4:761-768, 2019. doi: 10.1039/ C9ME00020H.

29. Matthew IJ Raybould, Claire Marks, Konrad Krawczyk, Bruck Taddese, Jaroslaw Nowak, Alan P Lewis, Alexander Bujotzek, Jiye Shi, and Charlotte M Deane. Five computational developability guidelines for therapeutic antibody profiling. Proc. Natl. Acad. Sci. USA, 116 (10):4025-4030, 2019. doi: 10.1073/pnas.1810576116.

30. Moriah Gidoni, Omri Snir, Ayelet Peres, Pazit Polak, Ida Lindeman, Ivana Mikocziova, Vikas Kumar Sarna, Knut EA Lundin, Christopher Clouser, Francois Vigneault, Andrew M Collins, Ludvig M Sollid, and Gur Yaari. Mosaic deletion patterns of the human antibody heavy chain gene locus shown by bayesian haplotyping. Nat. Commun., 10:628, 2019. doi: 10.1038/s41467-019-08489-3.

31. Benjamin North, Andreas Lehmann, and Dunbrack Jr. Roland L. A new clustering of antibody CDR loop conformations. J. Mol. Biol., 406(2):228-256, 2011. doi: 10.1016/j.jmb. 2010.10.030.

32. Veronica Morea, Anna Tramontano, Mauro Rustici, Cyrus Chothia, and Arthur M Lesk. Conformations of the third hypervariable region in the VH domain of immunoglobulins. J. Mol. Biol., 275(2):269-294, 1998. doi: 10.1006/jmbi.1997.1442.

33. Daisuke Kuroda, Hiroki Shirai, Masato Kobori, and Haruki Nakamura. Structural classification of CDR-H3 revisited: a lesson in antibody modeling. Proteins, 73(3):608-620, 2008. doi: $10.1002 /$ prot.22087.

34. Matthew IJ Raybould, Claire Marks, Alan P Lewis, Jiye Shi, Alexander Bujotzek, and Bruck Taddese. Thera-SAbDab: the Therapeutic Structural Antibody Database. Nucleic Acids Res., 48(D1):D383-D388, 2020. doi: 10.1093/nar/gkz827.

35. James Dunbar and Charlotte M Deane. ANARCI: antigen receptor numbering and receptor classification. Bioinformatics, 32(2):298-300, 2016. doi: 10.1093/bioinformatics/btv552.

36. Marie-Paule Lefranc, Christelle Pommié, Manuel Ruiz, Véronique Giudicelli, Elodie Foulquier, Lisa Truong, Valérie Thouvenin-Contet, and Gérard Lefranc. IMGT unique numbering for immunoglobulin and $\mathrm{T}$ cell receptor variable domains and Ig superfamily V-like domains. Dev. Comp. Immunol., 27(1):55-77, 2003. doi: 10.1016/S0145-305X(02)00039-3. 37. Yoonjoo Choi and Charlotte $M$ Deane. Accurate loop structure prediction using a database search algorithm. Proteins, 78(6):1431-1440, 2010. doi: 10.1002/prot.22658.

38. Yoonjoo Choi and Charlotte M Deane. Predicting antibody complementarity determining region structures without classification. Mol. BioSyst., 7(12):3327-3334, 2011. doi: 10. 1039/C1MB05223C

39. Limin Fu, Beifang Niu, Zhengwei Zhu, Sitao Wu, and Weizhong Li. CD-HIT: accelerated for clustering the next-generation sequencing data. Bioinformatics, 28(23):3150-3152, 2012. doi: 10.1093/bioinformatics/bts565. 